\title{
Sensitivity Study for the Optimization of the Viewing Chord Arrangement of the ITER Poloidal Polarimeter
}

\author{
Taiki YAMAGUCHI, Yasunori KAWANO and Yoshinori KUSAMA \\ Japan Atomic Energy Agency, Naka, Ibaraki 801-1, Japan
}

(Received 4 December 2006 / Accepted 4 April 2007)

\begin{abstract}
In order to optimize the viewing chord arrangement of the poloidal polarimeter in International Thermonuclear Experimental Reactor (ITER), we studied the sensitivity between the toroidal current profile and the viewing chord position. The current profile is scanned around the ITER operation scenario which is estimated as one of burning plasma operations. The sensitive viewing chords to distinguish changes of the central toroidal current density, the finite beta effect and the magnetic shear, are shown. The physical mechanisms of those sensitivities are also shown.
\end{abstract}

(C) 2007 The Japan Society of Plasma Science and Nuclear Fusion Research

Keywords: poloidal polarimeter, toroidal current profile, sensitivity study, viewing chord arrangement, magnetohydrodynamic equilibrium reconstruction, TOSCA, ITER

DOI: $10.1585 /$ pfr.2.S1112

\section{Introduction}

The poloidal polarimeter will be installed in the International Thermonuclear Experimental Reactor (ITER) to measure the profile of the toroidal current (safety factor) in the core region. The far-infrared laser beams (the wavelength is $118 \mu \mathrm{m}$ ) passing through the plasma are reflected back along the same path by retroreflectors. The Faraday rotation angle of the polarization plane of each beam is induced by the poloidal field inside the plasma. The number of viewing chords is restricted to about 15 channels because of the geometric capacity of port plugs [1]. Therefore the consideration of the optimum viewing chord arrangement is important. There are two points of views for the optimization. One is an accurate identification of the toroidal current profile. The first priority is the identification of the toroidal current profile in ITER design scenarios and the identification of the change around the scenarios with the required resolution. The second priority is the detection of the advanced physical phenomena like the current hole, the edge localized mode (ELM) and the disruption. Another point of view is the satisfaction of the engineering requirements. The detailed considerations of the optical system and components inside port plugs and inside the vacuum vessel, are necessary. It is out of scope of this paper.

The quantitative accuracy of the identified toroidal current profile can be evaluated by the Magnetohydrodynamic (MHD) equilibrium reconstruction. The accuracy of the toroidal current profile was evaluated in the previous work [1]. But the dependence of the viewing chord arrangement on the accuracy has not been considered yet. The evaluations using all combinations of viewing chords are actually impossible. Therefore the understanding the sensitive viewing chords to the change of the toroidal cur-

author's e-mail: yamaguchi.taiki@jaea.go.jp rent profile around the estimated toroidal current profile, is important as the first step. In this step, the physical mechanisms of the sensitivities should be also understood. The second step is the evaluation of the quantitative accuracy of the toroidal current profiles using the MHD equilibrium reconstruction method based on the knowledge given by the first step. This paper focuses on the first step. The toroidal current profile in one of ITER design scenarios is used as the estimated current profile.

\section{Candidates of Viewing Chord Ge- ometry and Calculation Method}

The equatorial (EQ) port and the upper (UP) port are used for the launching of the laser beam and the detections of the Faraday rotation angle [1]. Figure 1 shows the

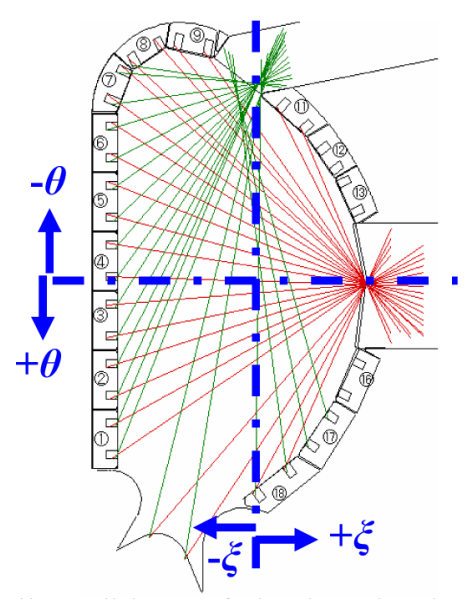

Fig. 1 All candidates of viewing chords of the ITER poloidal polarimeter. 
poloidal cross section of the ITER with all candidates of viewing chords. The viewing chord arrangement is based on the positions of retroreflectors, which are assumed inside the remote handling slots of the blanket modules in this study, and possible first mirror positions. 45 viewing chords are picked up. 9 channels for EQ port and 6 channels for UP port were proposed in the previous works [1] because of the geometric capacity of port plugs. Therefore the optimum selection is necessary.

The Faraday rotation angle $F$ is shown as the following equation:

$$
\begin{aligned}
F= & \frac{e^{3} \lambda^{2}}{8 \pi^{2} \varepsilon_{0} m_{e}^{2} c^{3}} \int n_{e} B_{\|} d l \\
= & \frac{e^{3} \lambda^{2}}{8 \pi^{2} \varepsilon_{0} m_{e}^{2} c^{3}} \int n_{e} B_{\|}^{p l} d l \\
& +\frac{e^{3} \lambda^{2}}{8 \pi^{2} \varepsilon_{0} m_{e}^{2} c^{3}} \int n_{e} B_{\|}^{v a c} d l,
\end{aligned}
$$

where $\lambda$ is the wavelength of the laser beam, $m_{e}$ is the mass of electron, $n_{e}$ is the electron density, $B_{\|}$is the magnetic field parallel to the direction of the viewing chord, $B_{\|}^{p l}$ is the magnetic field due to the plasma current and $B_{\|}^{\text {vac }}$ is one due to the external coils. The first term and the second term correspond to the Faraday rotation angles due to the plasma current and the external coils, respectively. We used the MHD equilibrium calculation code TOSCA [2] which has been used for the considerations of the operation scenario in ITER. The Faraday rotation angle can be calculated from the magnetic field distribution obtained as the result of TOSCA, the electron density profile and Eq. (1). Although the Faraday rotation angle is affected by the electron density, we assumed the fixed electron density profile as $n_{e}=10^{19}+10^{20}\left(1-\rho^{10}\right) \mathrm{m}^{-3}$ with $\rho$ the normalized minor radius, so that the sensitivity only of the toroidal current profile can be known. In ITER, $n_{e}$ can be measured by the toroidal interferometer/polarimeter system, the Thomson scattering system and/or the measurement of the Cotton-Mouton effect in the poloidal polarimeter.

\section{Sensitivity Study}

Figure 2 shows the calculated results of TOSCA. The central toroidal current density, $J_{0}$, is scanned. The net toroidal current $I_{p}$, the position and the shape of last closed flux surface (LCFS), the poloidal beta value $\beta_{p}$ and the pressure profile are fixed. Those fixed parameters and one of the toroidal current profiles (bold line in Fig. 2) are same as ITER design scenario II at the start of burn (SOB) phase. Figure 3 shows the Faraday rotation angle on viewing chords of the EQ port due to the plasma current, $F^{p l}$, which corresponds the first term of Eq. (1). The current profiles shown in Fig. 2 are used for the calculations. The calculations were done for single passage through the plasma. The definition of the viewing angle $\theta$ is shown in Fig. 1. The slope of $F^{p l}$ across the point of $F^{p l}=0$ is defined by
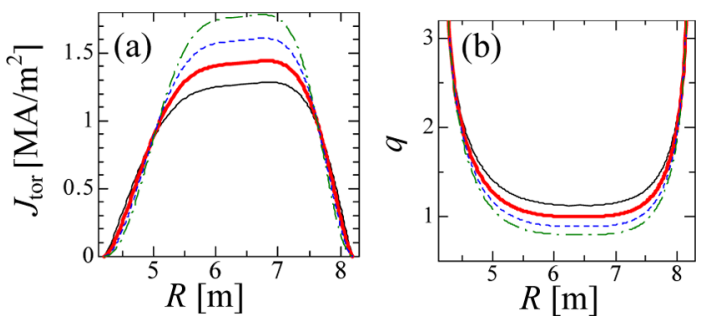

Fig. 2 The profiles of (a) the toroidal current and (b) the safety factor $q$ in case of $J_{0}$ scan, which are calculated by TOSCA.

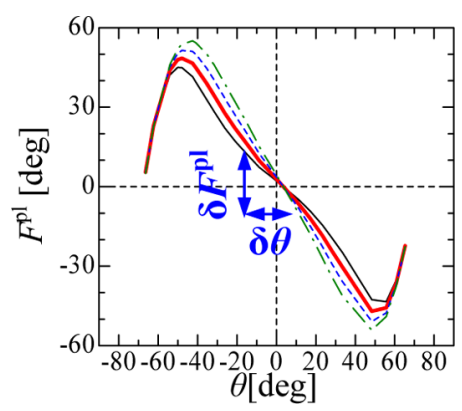

Fig. 3 Faraday rotation profiles which is induced by the toroidal current only.

$\delta F^{p l} / \delta \theta$, where $\delta F^{p l}$ is a difference between $F^{p l}$ of viewing chords of the EQ port at $\theta=-15 \mathrm{deg}$ and one at $11 \mathrm{deg}$. In the previous works, it was reported that (i) the relationship between the reciprocal of the central safety factor $q_{0}^{-1}$ and the slope of the $F$ is linear and (ii) the dependence of the toroidal current profile on $F$ is weak [3,4]. However both of $F$ and $q_{0}^{-1}$ include not only the magnetic field due to the toroidal current but also one due to external coils. Therefore the external magnetic field effect can be thought as the reason of the weak dependence of the toroidal current profile. The sensitivity of $F^{p l}$ is important for the optimization of the viewing chord arrangement because the magnetic field due to external coils can be easily given. So we evaluated the relationship between $J_{0}$ and the slope of $F^{p l}$. The result is shown in Fig. 4 in which conditions are same as ones for Fig. 2. The clear dependence can be seen. Nowadays polarimeters are able to resolve the Faraday rotation angle of $0.1 \mathrm{deg}$ [4]. This corresponds to $\delta F^{p l} / \delta \theta=0.0038$ as the slope. From Fig. $4, \delta F^{p l} / \delta \theta=0.013$ is enough for $\Delta J_{0}=1[\%]$. Therefore it can be said with good reliability that the central viewing chords of the EQ port are sensitive to the $J_{0}$. In those analyses, the sensitivity of $I_{p}$ was not discussed because $F^{p l}$ is proportional to $I_{p}$.

Next, the sensitivity of the finite beta effect is studied. The Pfirsch-Schlüter (P.S.) current is induced by the finite beta effect. The toroidal current without the P.S. current is fixed in this analysis. $I_{p}$ and the position and the shape of LCFS are also fixed. The poloidal beta value $\beta_{p}$ is scanned, i.e. $0.05,0.3,0.65$ and 0.8 . The dependence of $\beta_{p}$ on the central slope of $F^{p l}$ in the EQ port is shown in 


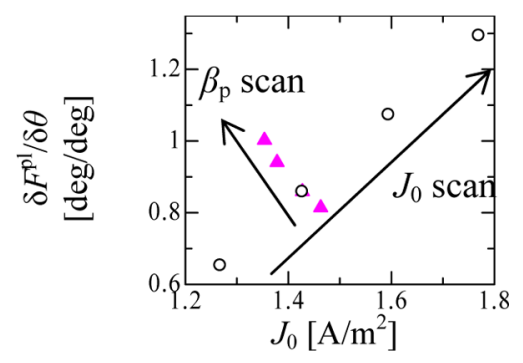

Fig. 4 The dependence of the toroidal current profile and $\beta_{p}$ on the central slope of $F^{p l}$ in the EQ port.

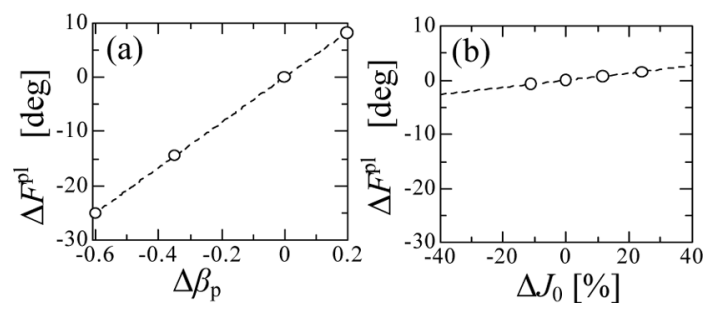

Fig. 5 The sensitivity of the central chord of the UP port to (a) $\beta_{p}$ and (b) $J_{0}$.

Fig. 4. The linear relationship between the $J_{0}$ and the slope is broken if $\beta_{p}$ changes. The additional information relating to $\beta_{p}$ is necessary because it is difficult to distinguish the change of $J_{0}$ from one of $\beta_{p}$ only by central viewing chords of the EQ port. The P.S. current induces the vertical magnetic field, which is denoted by $B_{z}^{P S}$. Then $B_{z}^{P S}$ induces the Shafranov shift. The Faraday rotation angle of each viewing chord is affected by $B_{z}^{P S}$ and the Shafranov shift. The P.S. current depends not only on the average beta value but also on the pressure profile. The diagnostics relating to the pressure profile, which measure the profiles of the electron density, the electron temperature, the ion temperature and the mean effective charge, can take the information of P.S. current. But the fewer diagnostics for the MHD reconstruction is attractive. Furthermore the neutral beam pressure and the pressure anisotropy depend on the P.S. current [5]. The measurements of those are difficult. The viewing chords of the UP port, which are sensitive to the P.S. current and not sensitive to the toroidal current profile, are needed. Figure 5 shows the dependence of $F^{p l}$ of the central chord in the UP port $(\xi \approx 0)$ on the changes of $\beta_{p}$ and $J_{0}$. The $\beta_{p}, J_{0}$ and $F^{p l}$ at ITER design scenario II (SOB) is used as the reference and the differences from that are shown. The change of $\Delta \beta_{p}=0.1$ induces $4.2 \mathrm{deg}$ of $\Delta F^{p l}$ although the change of $\Delta J_{0}=10 \%$ induces only $0.7 \mathrm{deg}$ of $\Delta F^{p l}$. Therefore it was found that the central chord of the UP port is sensitive to $\beta_{p}$ and less sensitive to $J_{0}$.

The plasmas in above analyses were the normal magnetic shear. The sensitivity of the hollow type toroidal current profiles, which construct the negative magnetic shear, was also studied. The example of that is shown in Fig. 6 (a)

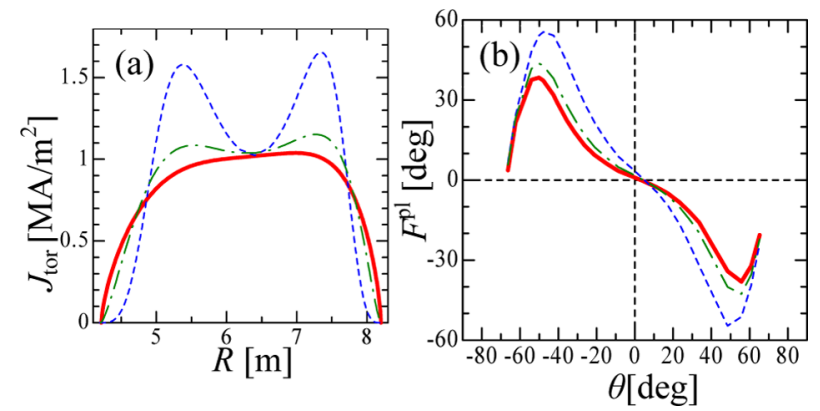

Fig. 6 The profiles of (a) the toroidal current and (b) $F^{p l}$ in case of $J_{\max }$ scan and fixed $J_{0}$.

Table 1 The comparison of the sensitivities of slopes of central and middle chords in the EQ port. The change of the slope of $F^{p l}$ [deg/deg] is shown.

\begin{tabular}{|c|c|c|}
\hline & $\begin{array}{l}\text { normal shear } \\
\text { (per } \Delta J_{0}=10 \\
\%)\end{array}$ & $\begin{array}{l}\text { negative shear } \\
\text { (per } \Delta\left(J_{0} / J_{\max }\right) \\
=0.1)\end{array}$ \\
\hline$\Delta$ (central slope) & $1.3 \times 10^{-1}$ & $1.1 \times 10^{-1}$ \\
\hline$\Delta$ (middle slope) & $4.0 \times 10^{-2}$ & $1.8 \times 10^{-1}$ \\
\hline
\end{tabular}

in which $I_{p}, \beta_{p}$, the position and the shape of LCFS and the pressure profile are same. Figure $6(\mathrm{~b})$ shows the $F^{p l}$ profile due to current profiles shown in Fig. 6(a). From Fig. 6, we can see that $F^{p l}$ of the central viewing chords change although $J_{0}$ does not change. The viewing chords of $\theta \sim \pm 30$ deg have relatively a long pass length in the region where the current density is near the maximum in the case of hollow profiles in Fig. 6. The viewing chords, which pass nearly $\theta \sim \pm 30 \mathrm{deg}$ (defined as middle region), may be useful to distinguish between the change of the maximum toroidal current density, $\Delta J_{\max }$, and $\Delta J_{0}$. It is estimated that the central viewing chords are more sensitive than the middle viewing chords when $J_{0}$ changes, on the other hand, the middle chords are more sensitive than the central chords when $J_{\max }$ changes. As the index, which can show the difference of the sensitivity between $\Delta J_{\text {max }}$ and $\Delta J_{0}$, we use the central slope, which is same definition as one in Fig. 3, and the middle slope, which is defined as the slope of $F^{p l}$ between $\theta=-35 \mathrm{deg}$ and $-26 \mathrm{deg}$. The sensitivities of those slopes to the toroidal current profiles are shown in Table. 1 which is based on Fig. 3 and Fig. 6. The change of the central slope is larger than one of middle slope when $J_{0}$ changes, whereas the change of the central slope is smaller than that when $J_{\max }$ changes. Therefore the combination of the central and the middle chords is useful to distinguish the change of $J_{0}$ and $J_{0} / J_{\text {max }}$. The weak sensitivities of the UP port to $J_{0} / J_{\max }$ was also found $\left(\Delta F^{p l}=0.58 \mathrm{deg} \operatorname{per} \Delta\left(J_{0} / J_{\max }\right)=0.1\right)$.

$\theta=-15$ and $11 \mathrm{deg}$ were used for the central slope of the EQ port. The smaller interval of them is better to detect the local toroidal current information near the plasma center. But the amplitudes of the signals and the amplitudes 


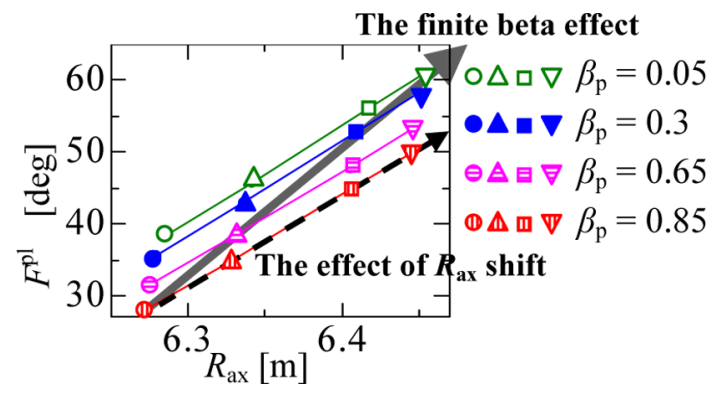

Fig. 7 The relationship between $R_{a x}$ and $F^{p l}$, where $R_{a x}$ shift is induced by the external coils or the finite beta effect.

of the difference between signals are smaller. $\theta=-35$ and $-26 \mathrm{deg}$ were used for the middle slope in the EQ port. They should be selected near the point of largest change of the slope of $F^{P L}$. The smaller interval is also better to distinguish the change of $J_{0}$ and $J_{0} / J_{\max }$ although the amplitude of the difference is smaller. It is necessary to evaluate the amplitudes of the signals based on small $I_{p}$, low $n_{e}$ and the broad toroidal current profiles.

\section{Discussion}

We discuss about the mechanism of the strong sensitivity shown in Fig. 5 (a). There are two possibilities as the reason, which are (i) $B_{z}^{P S}$ affects directly the central viewing chord in the UP port and (ii) the relative position between the viewing chord and the magnetic axis, $R_{a x}$, are changed by the Shafranov shift. It is interesting to compare the magnitude of those effects. For the comparison, two MHD equilibria, where the magnetic surface distributions are same (the effects of (ii) are same) but the beta values are different (the effects of (i) are different), are necessary. But those equilibria are difficult to make because of the Shafranov shift. Therefore we assume the effect of $R_{a x}$ shift by external coil currents changing as the effect of (ii), although that is rough estimation. The reason it is not exact estimation is that the $\Delta R_{a x}$ is different with the shift of the geometric center of LCFS, $\Delta R_{0}$, in case of the beta value increases, whereas $\Delta R_{a x}$ and $\Delta R_{0}$ are almost same in case of external coil currents changing. Figure 7 shows the relationship between $R_{a x}$ and $F^{p l}$, in which $R_{a x}$ shift is induced by the external coils and the finite beta effect. Colors mean the difference of $\beta_{p}$ and the shapes of symbols show the difference of $R_{a x}$ shift by external coils. The change of $\Delta \beta_{p}=0.1$ induces $\Delta F^{p l} \approx 4 \mathrm{deg}$, i.e. it is the effect of (i) and (ii), and $\Delta R_{a x} \approx 0.02 \mathrm{~m}$. The change of $F^{p l}$ is about $3 \mathrm{deg}$ if $\Delta R_{a x} \approx 0.02 \mathrm{~m}$ is induced by external coil's current, i.e. it is assumed as the effect of (ii). Therefore, from this analysis, it is thought that the effect of (ii) is much larger than one of (i).

\section{Future Plans}

The evaluation of the signal amplitude (see in Sec. 3) is the future work. The considerations of detailed positions of viewing chords in the other operation scenarios and in the advanced physical phenomena (see Sec. 1), are also future works although the mechanisms of sensitivities were known in this study.

MHD equilibrium reconstruction is also the future work. We are planning to develop the new reconstruction method. Most of reconstruction methods assume the GradShafranov equation. The reconstruction method, which does not assume the Grad-Shafranov equation, has been developed recently [6]. This method uses the magnetic diagnostics data and the constrainer of $\boldsymbol{J} \times \boldsymbol{B}=\boldsymbol{\nabla} p$. The poloidal polarimeter data has not been used. Our proposition is that the poloidal polarimeter data is used instead of the assumption of $\boldsymbol{J} \times \boldsymbol{B}=\boldsymbol{\nabla} p$. Therefore accurate reconstruction is expected on the conditions where $\boldsymbol{J} \times \boldsymbol{B}=\boldsymbol{\nabla} p$ does not stand up, for example, the highly anisotropic pressure plasma.

As optical arrangement inside the port plug, one viewing chord passes through one vacuum window in the present concept [1]. The alternative concept was also proposed, in which plural viewing chords can pass through one vacuum window, for a relaxation of the space limitation at the back plate of the port plug, a reduction of the vacuum leakage risk and a low cost [7]. The assessment of this concept is also the future work.

\section{Summary}

The following sensitivities are found with the physical mechanisms. It was shown with a good reliability that central viewing chords of the EQ port are sensitive to $J_{0}$ by removing the effect of external coils. The central viewing chord of the UP port is sensitive to the finite beta effect, moreover not sensitive to $J_{0}$ and $J_{0} / J_{\max }$. The combination of the central and the middle viewing chords of the EQ port, is useful to distinguish the changes of $J_{0}$ and $J_{0} / J_{\max }$. Those sensitivities and physical mechanisms are useful to the sensitivity studies of other toroidal current profiles and MHD equilibrium reconstruction, which are future works.

\section{Acknowledgement}

The authors would like to express their thanks to Drs M. Sugihara and H. Fujieda for useful discussion about the MHD equilibrium calculations.

[1] A.J.H. Donné et al., Rev. Sci. Instrum 75, 4694 (2004).

[2] H. Fujieda et al., JAERI-M 08-256, (JAERI, 1996).

[3] A.J.H. Donné et al., in Diagnostics for Experimental Thermonuclear Fusion Reactors 2, edited by P.E. Stott et al. (Plenum, New York, 1998) p. 203.

[4] C. Nieswand, in Diagnostics for Experimental Thermonuclear Fusion Reactors 2 , edited by P.E. Stott et al. (Plenum, New York, 1998) p. 213.

[5] T. Yamaguchi et al., Nucl. Fusion 45, L33 (2005).

[6] K. Kurihara et al., Fusion Eng. Des. 72, 527 (2005).

[7] Y. Kawano et al., 23rd Annual meeting of Japan Society of Plasma Science and Nuclear Fusion Research, 29aC04P (2006). 